\title{
Numerical Simulation of Turbulent Flow in Concentric Annuli
}

\author{
Bendiks Jan Boersma • Wim-Paul Breugem
}

Received: 29 December 2009 / Accepted: 26 August 2010 / Published online: 14 September 2010

(C) The Author(s) 2010. This article is published with open access at Springerlink.com

\begin{abstract}
In this paper we consider a fully developed turbulent flow in a round pipe with a small inner annulus. The diameter of the inner annulus is less than $10 \%$ of the diameter of the outer pipe. As a consequence, the surface area of the inner pipe compared to the outer pipe is small. The friction exerted by the wall on the flow is proportional to the surface area and the wall shear stress. Due to the small surface area of the inner annulus the additional stress on the flow due to the presence of the annulus may expected to be negligible. However, it will be shown that the inner annulus drastically changes the flow patterns and gives rise to unexpected scaling properties. In previous studies (Chung et al., Int J Heat Fluid Flow 23:426-440, 2002; Churchill and Chan, AIChE J 41:2513-2521, 1995) it was argued that radial position of the point of zero shear stress does not coincide with the radial location of the point of maximum axial velocity. In our direct numerical simulations we observe a coincidence of these points within the numerical accuracy of our model. It is shown that the velocity profile close to the inner annulus is logarithmic.
\end{abstract}

Keywords Turbulence $\cdot$ Pipe flow $\cdot$ DNS

\section{Introduction}

Turbulent pipe flow is one of the most studied turbulent flows. In this paper we will investigate the effect of a small inner annulus with negligible surface area on the flow and turbulent quantities in the pipe. It is shown that this inner annulus has large effects on the flow field. Although there are several applications for this type of flow such as oil drilling [8] or heat exchangers [2], our interest in this flow is not driven by the applications, but by the observation that the total (shear) stress is a

B. J. Boersma $(\bowtie) \cdot$ W.-P. Breugem

Department of Process and Energy, Delft University of Technology,

Leeghwaterstraat 44, 2628 CA Delft, The Netherlands

e-mail: b.j.boersma@tudelft.nl 
non-linear function of the distance to the wall. This is in contrast to almost all pressure driven channel and pipe flows, where in general linear total shear stress profiles are observed.

In the past annular turbulent pipe flow with an inner annulus has been studied quite extensively by Brighton and Jones [5], Quarmby [6] and Rehme [3]. Most of this work focuses on the determination of the friction factor and on the radial location of the maximum streamwize velocity. In the paper of Rehme [3] it is argued that the radial position for which the maximum axial velocity is obtained does not coincide with the position for which the Reynolds shear stress becomes zero. Churchill and co-workers $[4,7]$ use this observation to argue that eddy viscosity models can not be used to predict this flow and that this observation has implications for other turbulent flows. More recently Chung et al. [2] used Direct Numerical Simulation to calculate the flow and heat transfer in annuli. They also observe a small difference in the radial position for which the mean velocity profile has a maximum and the point where the Reynolds shear stress is zero. It should be noted that the radial resolution of the simulations performed by Chung et al. [2] is rather poor. Kaneda et al. [4] model the flow between the concentric cylinders based on the assumption that the point where the Reynolds shear stress is zero and the maximum for the mean axial velocity do not coincide. At present there seems to be a consensus in the literature for the non-coincidence of the location of the zero shear stress and mean velocity in pipe flow with an inner annulus. In the present paper we will use a well resolved direct numerical simulation to study the flow between two concentric cylinders. In our simulations we do not observe a non-coincidence between the point where the Reynolds shear stress is zero and the location of the maximum mean velocity. It is argued that the experimental observations of Rehme [3] are biased by measurement errors or end effects in the experimental setup. The work of Churchill and co-workers is based on these observations.

The organization of the paper is as following. In Section 2 we give the governing equations for the flow, in Section 3 we will discuss the numerical model which has been used to solve the equations. In Section 4 we show results for the shear stress profiles and the radial position of the maximum mean velocity. In Section 5 mean and rms velocity profiles are presented. Next in Section 6 we show results for the case of negligible shear stress on the outer pipe, and finally discussions and conclusions are given in Section 7.

\section{Geometry and Governing Equations}

In this paper we will consider the flow in a fully developed cylindrical pipe with a thin inner annulus. A sketch of the geometry is shown in Fig. 1. The radius of the inner annulus will be denoted by $r_{a}$ and the radius of the outer annulus by $R$. Throughout the paper we will assume that $r_{a} / R \ll 1$ and that the fluid is incompressible.

The time and spatially averaged flow in the pipe is described by the NavierStokes equations in cylindrical coordinates. The axial component of the NavierStokes equation reads:

$$
\frac{\partial w}{\partial t}+\frac{1}{r} \frac{\partial}{\partial r} r u w+\frac{1}{r} \frac{\partial}{\partial \theta} v w+\frac{\partial}{\partial z} w w=-\frac{1}{\rho} \frac{\partial p}{\partial z}+v\left[\frac{1}{r} \frac{\partial}{\partial r}\left(r \frac{\partial w}{\partial r}\right)+\frac{1}{r^{2}} \frac{\partial^{2} w}{\partial \theta^{2}}+\frac{\partial^{2} w}{\partial z^{2}}\right] .
$$


Fig. 1 A sketch of the geometry

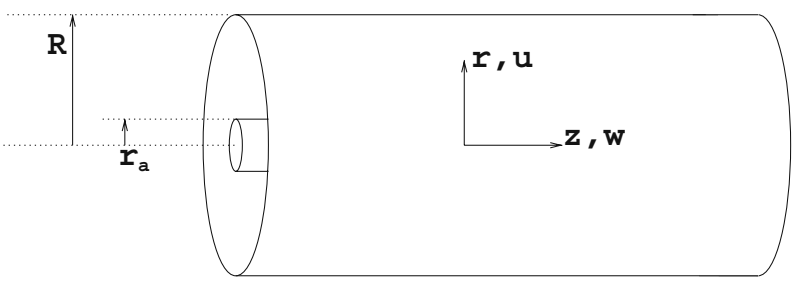

L

In which $u, v, w$ are the velocity components in the radial, circumferential and axial directions, $\rho$ the density, $p$ is the pressure, and $v$ the kinematic viscosity. If we apply a Reynolds averaging procedure and assume that the flow is fully developed in the axial direction, (statistically) axi-symmetric and statistically steady, equation (1) reduces to:

$$
\frac{1}{r} \frac{\partial}{\partial r}\left(r \overline{u^{\prime} w^{\prime}}-v r \frac{\partial \bar{w}}{\partial r}\right)=-\frac{1}{\rho} \frac{\partial \bar{p}}{\partial z} .
$$

In which $\overline{u^{\prime} w^{\prime}}$ is the Reynolds shear stress, $\bar{w}$ the Reynolds averaged axial velocity profile, and $\bar{p}$ the Reynolds averaged pressure. With help of the radial component of the Reynolds averaged Navier-Stokes equations (not shown here) it can be shown that $\partial \bar{p} / \partial z$ is not a function of $r$ and equation (2) can be easily integrated. The result reads

$$
\left(r \overline{u^{\prime} w^{\prime}}-v r \frac{\partial \bar{w}}{\partial r}\right)=-\frac{r^{2}}{2} \frac{1}{\rho} \frac{\partial \bar{p}}{\partial z}+C
$$

Where $C$ is an integration constant. We can also rewrite equation (3) as

$$
\overline{u^{\prime} w^{\prime}}-v \frac{\partial \bar{w}}{\partial r}=-\frac{r}{2} \frac{1}{\rho} \frac{\partial \bar{p}}{\partial z}+\frac{C}{r}
$$

The actual value of $C$ can be expressed as a function of the wall shear stress on the annulus and outer cylinder, denoted by $\tau_{a}$ and $\tau_{o}$ respectively:

$$
C=-\frac{1}{\rho} \frac{\tau_{o} r_{a}-\tau_{a} R}{r_{a} / R-R / r_{a}}, \quad \text { with } \quad \tau_{a}=\left.\rho v \frac{\partial \bar{w}}{\partial r}\right|_{r=r_{a}}, \tau_{o}=\left.\rho v \frac{\partial \bar{w}}{\partial r}\right|_{r=R},
$$

If we take the limit $r_{a} \rightarrow 0$ the value of $C \rightarrow 0$. The assumption that the flow is statistically steady implies that the pressure gradient in equation (4) should be balanced by the wall shear stress on the inner and outer cylinder, this results in:

$$
\frac{1}{2} \frac{\partial \bar{p}}{\partial z}=\frac{\tau_{o} R-\tau_{a} r_{a}}{R^{2}-r_{a}^{2}}
$$

With help of this relation and the expression we found for $C$ we can rewrite equation (4) as

$$
\overline{u^{\prime} w^{\prime}}-v \frac{\partial \bar{w}}{\partial r}=-\frac{1}{\rho\left(R^{2}-r_{a}^{2}\right)}\left(r\left[\tau_{o} R-\tau_{a} r_{a}\right]-\frac{r_{a} R}{r}\left[\tau_{o} r_{a}-\tau_{a} R\right]\right)
$$


For scaling purposes it is useful to introduce a velocity scale. Here we will use velocity $U_{*}$ which is based on the applied (external) pressure gradient

$$
U_{*}=\sqrt{-\frac{R}{2 \rho} \frac{\partial \bar{p}}{\partial z}}
$$

If $r_{a} \rightarrow 0$ the velocity scale $U_{*}$ is equal to the classical friction velocity in a standard pipe flow.

\section{Direct Numerical Simulations and Simulation Details}

For the direct numerical solution of the Navier-Stokes equations we use the numerical model developed by Eggels et al. [1] for cylindrical pipe flow. In this model the Navier-Stokes equations, formulated in a cylindrical coordinate system, are discretized with a second order finite volume method. The time integration has been performed with a second order Adams-Bashforth method. Conservation of mass is ensured with a pressure correction method. Due to the presence of the small inner annulus no singularity is present in the computational domain which would be the case if we would include the centre region. Therefore, there is no need for special treatment near $r=r_{a}$. At the in and outflow section periodic conditions are used. No slip conditions are used on both the inner and outer wall. The length of the computational domain is $10 R$ with $R$ the radius of the outer cylinder. This domain length is the same as was used by Eggels et al. [1] in which it was shown that this domain length was sufficient to obtain negligible autocorrelation function of the velocity components halfway the domain. The ratio $r_{a} / R$ is equal to $0.02,0.04$ or 0.10 . The Reynolds number based on the mean friction velocity and pipe diameter $R e=2 U_{*} R / v$ is equal to 600 . For the case with $r_{a} / R=0.10$ we have also performed a simulation with a slightly higher Reynolds number $R e=2 U_{*} R / v=900$. This has been done to check the Reynolds dependency of the flow. The simulations are performed on a uniform mesh of $192 \times 192 \times 384$ points in the radial, circumferential and axial direction respectively. To check the accuracy of the simulations also simulations on a finger mesh of $384 \times 320 \times 512$ have been performed. The grid spacing in plus units for the various simulations is reported in Table 1 . When we compute the results obtained with the different grid sizes we observe no noticeable difference between the results. Most of the results presented in here are obtained with the resolution of $192 \times 192 \times 384$ points. The flow in our simulations is driven

Table 1 The grid spacing in plus units, based on the mean friction velocity $U_{*}$

\begin{tabular}{llll}
\hline & $r^{+}$ & $r_{a} \Delta \theta^{+}$ & $z^{+}$ \\
\hline$r_{/} R=0.02(192 \times 192 \times 384)$ & $1.5(2.6)$ & 9.8 & 7.8 \\
$r_{a} / R=0.04(192 \times 192 \times 384)$ & $1.4(2.2)$ & 9.8 & 7.8 \\
$r_{a} / R=0.10(192 \times 192 \times 384)$ & $1.3(1.5)$ & 9.8 & 7.8 \\
$r_{a} / R=0.02(384 \times 320 \times 512)$ & $0.8(1.3)$ & 5.9 & 5.6 \\
$r_{a} / R=0.04(384 \times 320 \times 512)$ & $0.7(1.1)$ & 5.9 & 5.6 \\
$r_{a} / R=0.10(384 \times 320 \times 512)$ & $0.7(0.8)$ & 5.9 & 5.6 \\
\hline
\end{tabular}

The value between brackets is the gridspacing close the inner annulus based on the local friction velocity 
Fig. 2 A slice in the $r-\theta$-plane. The contour denotes the axial velocity scaled with $U_{*}$ and the vectors the flow in the $r-\theta$-plane (every fourth vector is shown), $r_{a} / R=0.02$

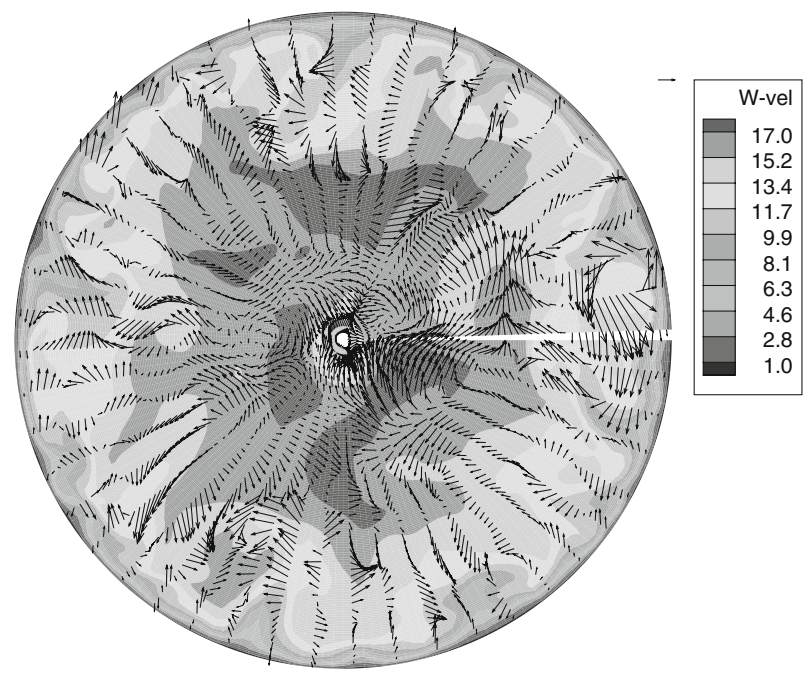

by a constant pressure gradient. The pressure gradient is given by equation (6) but the values of $\tau_{o}$ and $\tau_{a}$ are not known a priori. We fix the value of pressure gradient as

$$
-\frac{1}{\rho} \frac{\partial \bar{p}}{\partial z}=\frac{2 U_{*}^{2}}{R}
$$

With this pressure gradient we expect that $\tau_{o}$ is $\mathcal{O}\left(\rho U_{*}^{2}\right)$. The actual values of $\tau_{o}$ and $\tau_{a}$ can be calculated from the simulation results. As initial conditions for the simulations we use a constant axial velocity $\bar{w}=18 U *$. On top of this velocity profile white noise random perturbations, with a maximum of $5 U_{*}$, are superimposed. The simulations are run sufficiently long (typically for $30 R / U_{*}$ ) to become independent of the initial conditions. After this initial period the simulations are continued for

Fig. 3 A slice in the $r-z$-plane. The contours of the circumferential velocity $v=v_{\theta}$ normalized with $U_{*}$ are shown, $r_{a} / R=0.02$

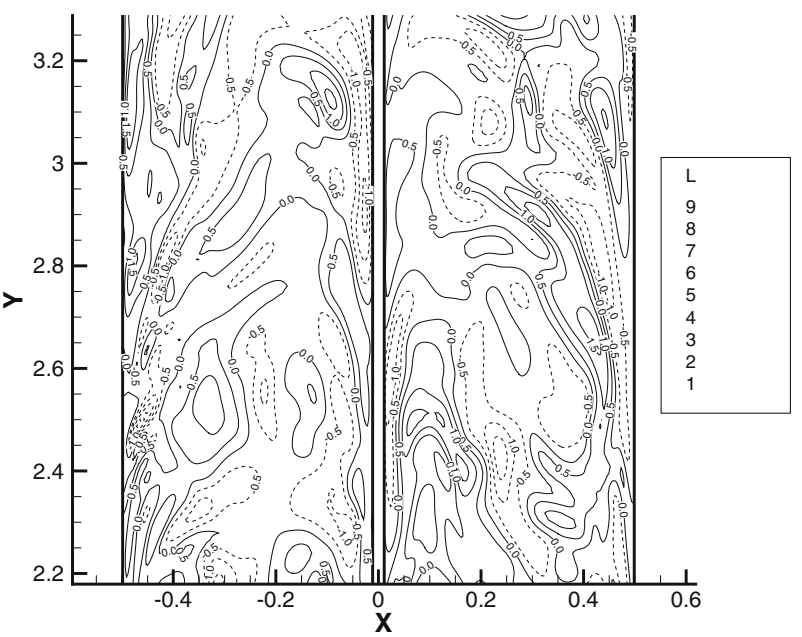


another $10 R / U_{*}$ over which 50 datafields equally separated in time are stored. The results presented in the following sections are based on the spatial and temporal averages over these 50 datafields. Most of the results presented in the following sections will be normalized with the mean friction velocity $U_{*}$ or with the local rescaled wall shear stresses $\sqrt{\tau_{a} / \rho}, \sqrt{-\tau_{o} / \rho}$.

An illustration of the simulated flow is given in Figs. 2 and 3 where we show the axial velocity component in the $r-\theta$-plane and the circumferential velocity component in the $r-z$ plane, for a flow with $r_{a} / R=0.02$.

\section{Reynolds Shear Stress Profiles and Velocity Maximum}

In this section we will present the Reynolds and total shear stress profiles and we will compare the radial position for which the total stress is zero with the radial position for which the axial velocity has a maximum. In Fig. 4, (left) we show the Reynolds shear stress profile $\overline{u^{\prime} w^{\prime}}$, normalized with $U_{*}^{2}$, for a standard pipe flow, see Eggels et al. [1] and for the flow with three different inner annuli, labeled with $r_{a} / R=0.02,0.04$ and 0.1 (the "l" denotes the result obtained with the low resolution, $192 \times 192 \times 384$ and the " $h$ " denotes the result obtained with the high resolution, $384 \times 320 \times 512$ ). Close to the outer wall the profiles are very similar. Close to the inner annulus the $1 / r$ behavior is clearly visible in the profiles. The effect of the different numerical resolution is very small. In Fig. 4, (right) we show the total stress, equation (4), $\overline{u^{\prime} w^{\prime}}-v \partial \bar{w} / \partial r$.

The numerical value of the constant $C$ appearing in equation (4) can easily be calculated using the values for the wall shear stress $\tau_{o}, \tau_{a}$ and equation (5). The numerical values for the constant $C$ and the values of $\tau_{o}$ and $\tau_{a}$ are given in Table 2 . In Fig. 5 we plot the total shear stress from the simulation with an annulus of $r_{a} / R=0.04$ together with the profile $-\frac{1}{2 \rho} r \partial \bar{p} / \partial z+C / r$. Clearly the agreement is excellent, which is a good indication that our results are statistically converged.

As mentioned in the introduction there is some discussion in the literature about the coincidence of the radial position where the shear stress is zero and the maximum of the axial velocity profile. In Table 2 we have given the radial locations for which
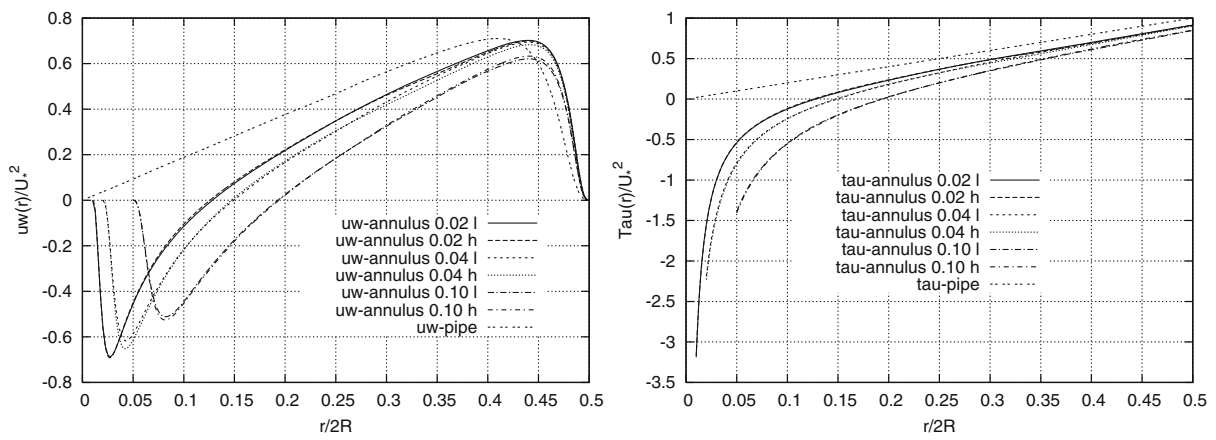

Fig. 4 The Reynolds stress (left) as a function of the radial coordinate for a standard pipe flow and the flow in annuli with $r_{a} / R=0.02, r_{a} / R=0.04, r_{a} / R=0.10$. Right the total shear stress in standard pipe flow and the flow in annuli 
Table 2 Simulation details

\begin{tabular}{lllll}
\hline$r_{a} / R$ & 0.02 & 0.04 & 0.1 & 0.1 \\
$R e_{b}$ & 8,900 & 8,910 & 9,270 & 13,940 \\
$C$ & -0.0318 & -0.0445 & -0.0744 & -0.0721 \\
$\langle r| \overline{u^{\prime} w^{\prime}}=0>$ & $0.126(0.126)$ & $0.149(0.147)$ & $0.193(0.194)$ & 0.190 \\
$<r \mid \bar{w}=w_{\max }>\left(=r_{m}\right)$ & $0.124(0.125)$ & $0.147(0.148)$ & $0.194(0.194)$ & 0.190 \\
$\left|\tau_{a}\right| /\left(\rho U_{*}^{2}\right)$ & $3.16(3.14)$ & $2.18(2.20)$ & $1.39(1.40)$ & 1.34 \\
$\left|\tau_{o}\right| /\left(\rho U_{*}^{2}\right)$ & $0.91(0.91)$ & $0.91(0.90)$ & $0.84(0.85)$ & 0.85 \\
\hline
\end{tabular}

The numbers between () are obtained with a resolution of $384 \times 320 \times 512$ points. $\left|\tau_{a}\right| /\left(\rho U_{*}^{2}\right)$ denotes the normalized wall shear stress at the annulus and $\left|\tau_{o}\right| /\left(\rho U_{*}^{2}\right)$ the normalized shear stress at the outer pipe wall

$r_{a}$ is the radius of the inner annulus

$R$ the radius of the outer annulus

$R e_{b}$ the Reynolds number based on the bulk flow

$C$ the constant in equation (4)

$<r \mid \overline{u^{\prime} w^{\prime}}=0>$ the radius for which the total shear stress is zero

$<r \mid w_{\max }>$ the radius at which the axial velocity reaches its maximum

we observed a zero Reynolds shear stress. Also we have included the radial position for which the velocity profile reaches its maximum. This radial position is obtained by numerically differentiating the axial velocity profile. With a resolution of 192 points in the radial direction we have a radial gridspacing $\Delta r \approx 0.005 R$. The differences between the radial positions for the zero shear stress and the maximum velocity are of the same order of the gridspacing and therefore we conclude that the radial positions for zero shear stress and maximum axial velocity coincide within the accuracy of the present direct numerical simulation. The difference in radial locations in the DNS performed by Chung et al. [2] is in our view caused by the rather poor numerical resolution (65 points) in the radial direction. From a theoretical point of view it is also unlikely that in a high Reynolds number flow a difference between the zero crossing of the Reynolds shear stress and the maximum axial velocity occurs. At the point in the flow for which the total shear stress is zero, equation (4) gives

$$
\overline{u^{\prime} w^{\prime}}=v \frac{\partial \bar{w}}{\partial r}
$$

Fig. 5 The total stress a function of the radial coordinate for the flow with the annulus, symbols DNS, line $-\frac{1}{2 \rho} r \partial \bar{p} / \partial z+C / r$ with $C$ as given in Table 2

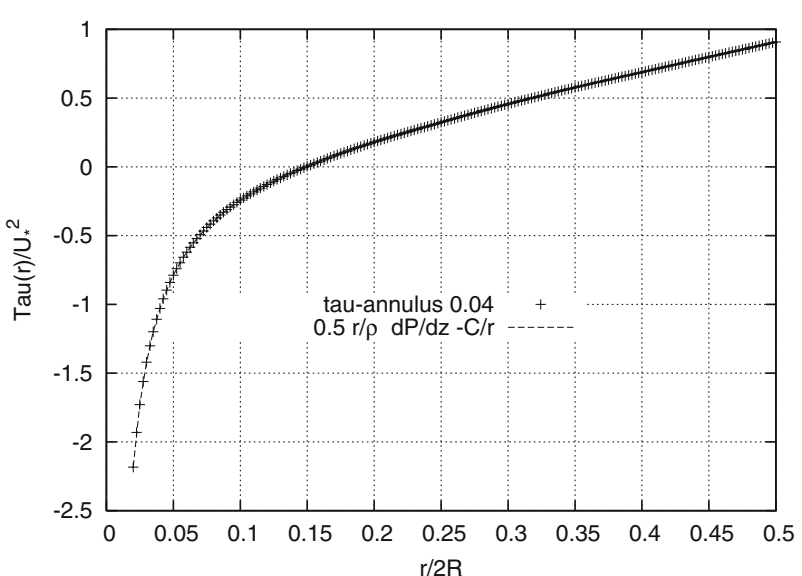


In principle $\overline{u^{\prime} w^{\prime}}$ does not have to be equal to zero. However in a high Reynolds number channel or pipe flow viscous effects are only important in the near wall region where the gradients in the mean velocity profile are very large. If we assume that the point where the total shear stress is zero is located sufficient away far from the wall viscous effects can be considered negligible and we can assume that $\overline{u^{\prime} w^{\prime}}=0$ at the point where the total shear stress is zero. For nearly parallel flows, such as for instance discussed by [10] additional terms may play a role and it will be possible that $\overline{u^{\prime} w^{\prime}} \neq 0$, but for the present flow $\overline{u^{\prime} w^{\prime}}$ should be zero, or at least $\overline{u^{\prime} w^{\prime}} / U_{*}^{2} \leq O\left(R e^{-1}\right)$. Unfortunately, we can not show from first principles that $\partial \bar{w} / \partial r$ is zero in the point with zero total stress, but the DNS results clearly show this.

\section{Mean Velocity Profiles}

In this section we will first give some standard closure approximations for wall bounded shear flows and will try to predict the mean turbulent velocity profile. Subsequently these findings will be compared with the results of the DNS.

In previous work by, Churchill and co-workers [7], it was argued that the use of eddy viscosity approaches for this flow is "futile" because there is a shift in the radial position between the point for zero shear stress and the maximum velocity profile. The results from our DNS do not support this statement and therefore we base our model on classical eddy viscosity assumptions. We will only consider the flow in the layer $r \leq r_{m}$, where $r_{m}$ is the radial location for which the axial velocity reaches its maximum (the layer $r \geq r_{m}$ scales as a classical pipe flow and results can be found elsewhere [12]).

\subsection{The viscosity dominated region}

In the region very close to the annulus $\left(r \approx r_{a}\right)$ it is assumed that the viscous stresses are much larger than the Reynolds shear stress, equation (7) reduces to:

$$
-v \frac{\partial \bar{w}}{\partial r}=-\frac{1}{\rho R^{2}}\left(r\left[\tau_{o} R-\tau_{a} r_{a}\right]-\frac{r_{a} R}{r}\left[\tau_{o} r_{a}-\tau_{a} R\right]\right) \approx-\frac{r_{a} \tau_{a}}{\rho r}, \text { if } r_{a} \ll R,
$$

After integration and applying the no-slip condition on the annulus, we find that the velocity profile for $r \approx r_{a}$ is given by

$$
\bar{w}(r)=\frac{r_{a} \tau_{a}}{\rho v} \log \left(\frac{r}{r_{a}}\right) .
$$

This solution is clearly different from the near wall solution for a standard wall bounded shear flow where the region close to the wall is linear.

\subsection{The Reynolds stress dominated region}

Away from the wall we assume that the Reynolds shear dominates the viscous stress and equation (7) reduces to

$$
-\overline{u^{\prime} w^{\prime}} \approx \frac{1}{\rho R^{2}}\left(r\left[\tau_{o} R-\tau_{a} r_{a}\right]-\frac{r_{a} R}{r}\left[\tau_{o} r_{a}-\tau_{a} R\right]\right) \approx \frac{1}{\rho}\left(\frac{\tau_{a} r_{a}}{r}+\frac{\tau_{o} r}{R}\right) \text { if } r_{a} \ll R
$$


Let us first consider the case $\tau_{o} r^{2} / \tau_{a} r_{a} R \ll 1$. In this case the right hand side of the equation above reduces to: $\tau_{a} r_{a} / \rho r$ In standard eddy viscosity approach the following closure for the Reynolds shear stress is used [12]:

$$
\overline{u^{\prime} w^{\prime}}=-v_{t} \frac{\partial \bar{w}}{\partial r}
$$

In which $v_{t}$ is the eddy viscosity. The eddy viscosity is in general modeled with a mixing length model:

$$
v_{t}=L^{2}\left|\frac{\partial \bar{w}}{\partial r}\right|
$$

Where $L$ is the length scale of the eddies. The size of the eddies scales with the distance to the wall, in this case $r-r_{a}$ and we can write

$$
v_{t}=\alpha^{2}\left(r-r_{a}\right)^{2}\left|\frac{\partial \bar{w}}{\partial r}\right|
$$

where $\alpha$ is a non-dimensional constant. Equation (7) can now be approximated as

$$
\alpha^{2}\left(r-r_{a}\right)^{2}\left|\frac{\partial \bar{w}}{\partial r}\right| \frac{\partial \bar{w}}{\partial r} \approx \frac{\tau_{a} r_{a}}{\rho r}
$$

The solution of this equation is given by

$$
\bar{w}(r)=\frac{2}{\alpha} \sqrt{\frac{\tau_{a}}{\rho}} \operatorname{arctanh}\left[\sqrt{\frac{r}{r_{a}}}\right]+\text { Const }
$$

This solution does not exist for $r>r_{a}$.

Another model for the eddy viscosity $v_{t}$ could be

$$
v_{t}=\alpha \sqrt{\frac{\tau_{a}}{\rho}}\left(r-r_{a}\right)
$$

with this model equation (7) can be approximated as

$$
\alpha \sqrt{\frac{\tau_{a}}{\rho}}\left(r-r_{a}\right) \frac{\partial \bar{w}}{\partial r} \approx \frac{\tau_{a} r_{a}}{\rho r}
$$

In this case the solution for the velocity profile $\bar{w}$ is given by

$$
\bar{w}=\sqrt{\frac{\tau_{a}}{\rho}} \frac{1}{\alpha} \log \left[\frac{r-r_{a}}{r}\right]+D_{1}
$$

Where $D_{1}$ is an integration constant. This solution does exist for values $r>r_{a}$.

If we consider the case $\tau_{o} r^{2} / \tau_{a} r_{a} R \gg 1$ we find the (classical) linear relation for the shear stress and the resulting velocity profile will be logarithmic, see for instance Pope [12].

\subsection{DNS results}

In this section we will present DNS results for the mean flow profiles and compare them with the approximations given before. The mean axial velocity profiles for standard pipe flow [1] and for the cases with different inner annuli are given in Fig. 6. All the results are scaled with the velocity scale $U_{*}$. The profiles close to 

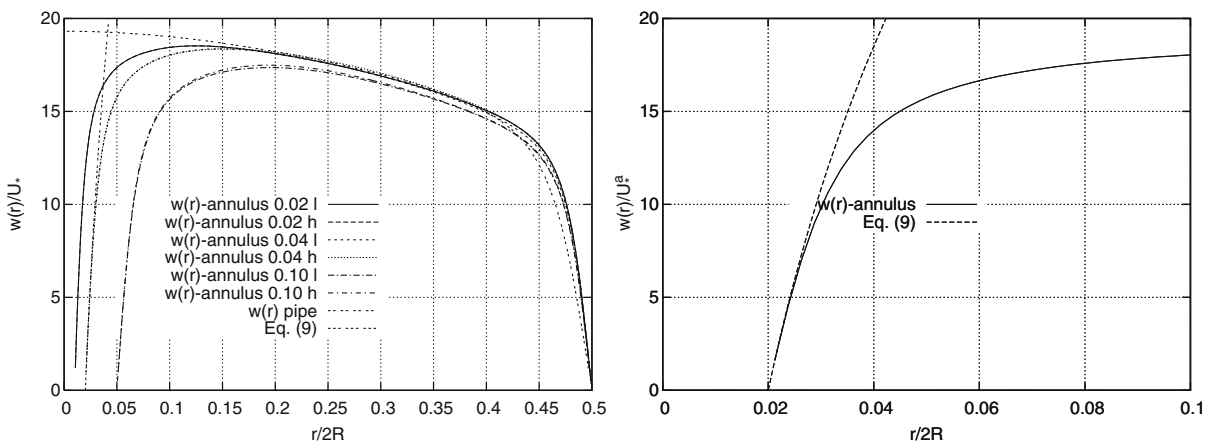

Fig. 6 Left: The mean velocity profiles normalized with $U_{*}$ as a function of the radial coordinate, for the standard pipe flow and annuli with $r_{a} / R=0.02, r_{a} / R=0.04$ and $r_{a} / R=0.10$. Right, the profile close to the inner annulus for the case $r_{a} / R=0.04$, together with the approximate solution given by equation (9)

the outer wall, i.e. near $R=0.5$ are nearly identical for both flows. The flow rate is clearly larger for the flow without the annulus, i.e. less friction results in a higher bulk velocity. The approximate solution given by equation (9), which is valid close to the inner annulus is also shown for the case $r_{a} / R=0.04$. As can be seen from the Fig. 6, equation (9) agrees nicely with the result of the DNS in the region $r \approx r_{a}$. From the literature, see for instance [3], it is known that the $r$ value $\left(r_{m}\right)$ for which the mean velocity reaches a maximum, is given by the following empirical relation

$$
\frac{r_{m}-r_{a}}{R-r_{m}}=\left(\frac{r_{a}}{R}\right)^{0.343}
$$

For our geometry with $r_{a} / R=0.02$ this gives a $r_{m} / R=0.11$. In the DNS we observe a slightly higher value $r_{m} / R \approx 0.13$. For $r_{a} / R=0.04$ the correlation gives $r_{m} / R=0.14$ and the DNS gives 0.15 , for $r_{a} / R=0.1$ we find $r_{m} / R=0.19$ and for the DNS also 0.19 . For small values of $r_{a} / R$ there is thus a difference between the results of the correlation and the DNS. For larger values of $r_{a} / R$ the agreement is excellent.

In Fig. 7 we show velocity profiles scaled with the local friction velocity. The flow close to the outer wall behaves as a standard pipe or channel flow. In the
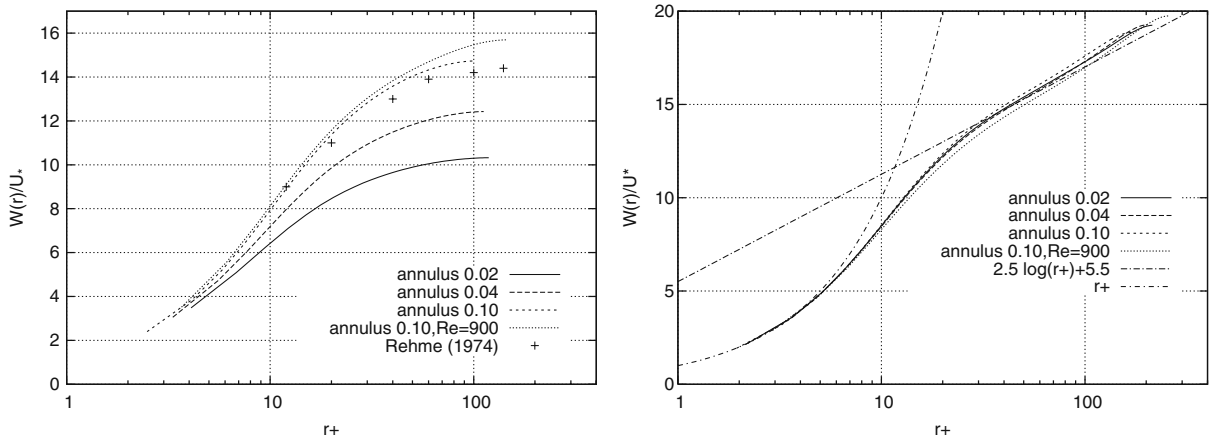

Fig. 7 The mean velocity profile scaled with the local friction velocity as a function of the distance to the wall. Left the profile close to the inner annulus, right the profiles close to the pipe wall 
Fig. 8 The mean velocity profiles and the profiles given by equation (12). The constants are obtained with a least square fit

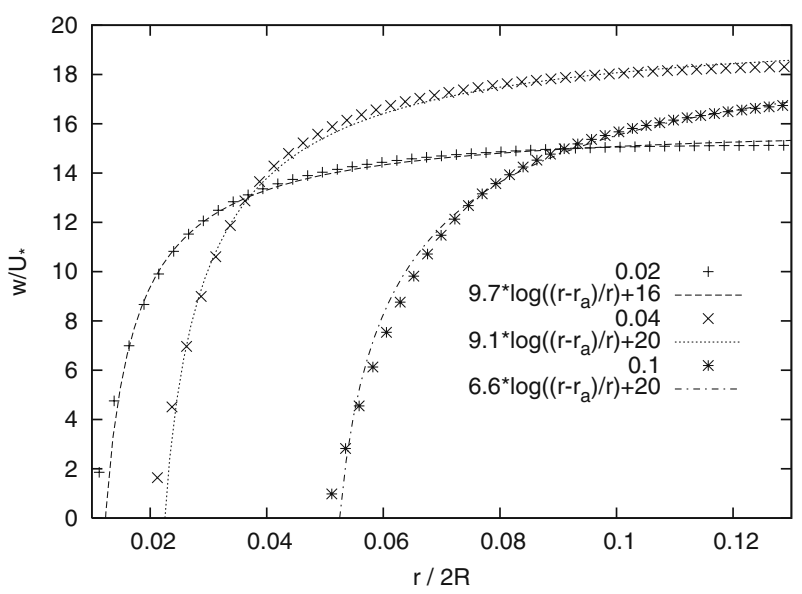

first 5 to 10 viscous wall units $\left(r^{+}=|R-r| \sqrt{-\tau_{o} / \rho} / \nu\right)$ the mean velocity profiles is approximately linear. For larger distance to the wall we observe a logarithmic region. The slope and the constant are similar to what is found for standard pipe flow $\bar{W}(r)=2.5 U_{*} \log \left(r U_{*} / v\right)+5.5$ (see for instance Eggels et al. [1]). The flow close to the inner annulus is completely different. Very close to the annulus, in the first 10 25 viscous units $\left(r^{+}=\left(r-r_{a}\right) \sqrt{\tau_{a} / \rho} / v\right)$ the profile is logarithmic, which agrees with the result given by equation (9). For comparison we have also included some of the experimental data reported by [3]. The agreement between the experimental data and simulation is reasonably good.

In Fig. 8 we show the axial velocity profiles together with the velocity profiles given by equation (12). The two constants in equation (12) are obtained with a least square fit. The values of the constants are given in the figure.

\subsection{Turbulence intensities}

In this section the turbulence intensities on the inner and outer wall will be presented. In Fig. 9 we show the turbulence intensities of the three velocity components,

Fig. 9 The rms profile of the three velocity components scaled with the mean friction velocity

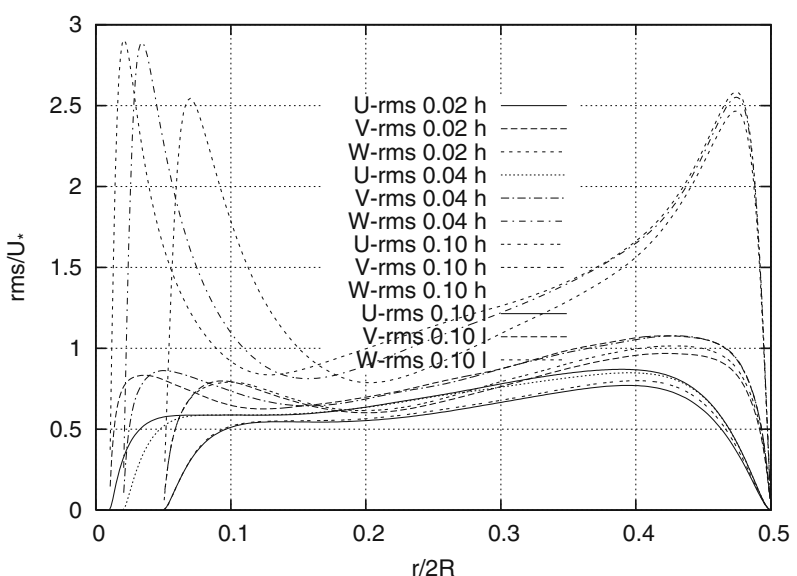



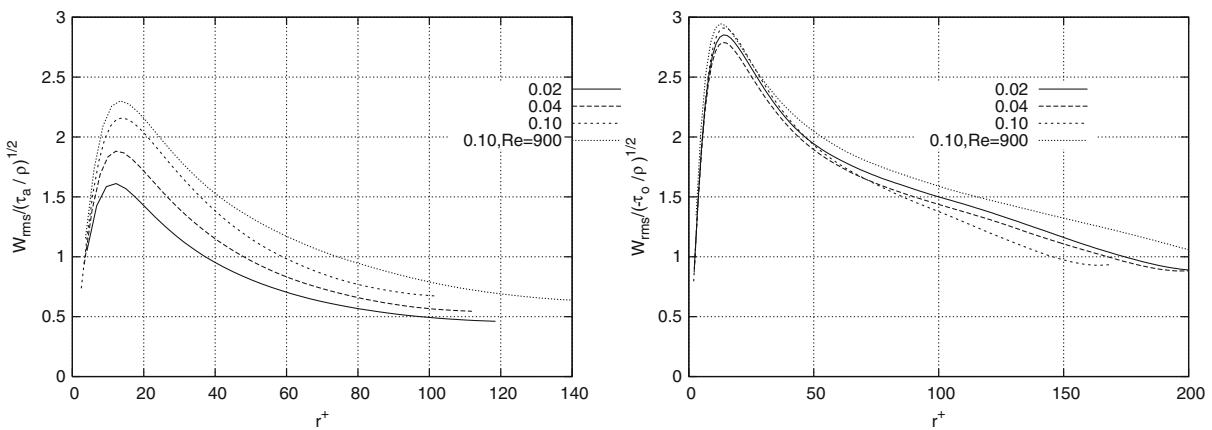

Fig. 10 The rms profile of the three velocity components plotted as a function of $r^{+}$, left inner wall $\left(r^{+}=\left(r-r_{a}\right) \sqrt{\tau_{a} / \rho} / \nu\right)$, right outer wall $\left(r^{+}=|R-r| \sqrt{\tau_{a} / \rho} / \nu\right.$. $)$

scaled with velocity scale $U_{*}$. In Fig. 10 we show the axial component of the rms profile scaled with the square root of the local wall shear stress and plotted as a function of $r^{+}$(defined in the caption of Fig. 10). The profiles at the outer wall collapse and the maximum is approximately 2.7 times the local friction velocity. This is a value which is observed in almost all wall bounded flows [9]. At the inner wall the peak of the axial rms clearly depends on the radius of the inner cylinder. This dependency was also observed by [11]. They give the following argument: In transversely curved flows there is a smaller surface area over which vorticity fluctuations can be generated relatively to the volume of turbulent flow supported. The annulus is not less efficient as a source of turbulent kinetic energy but is has to support a larger area. Furthermore there is a clear Reynolds number effect visible, between the two simulations with an annulus ratio of 0.1 . This effect is absent at the outer wall.

\section{Flow Without Outer Pipe}

In the right hand side of equation (7) there is always a $r^{-1}$ and a $r$ behavior. The $r^{-1}$ behavior is due to the inner cylinder and the $r$ behavior due to the outer cylinder. In this section we will present some results obtained from simulations without an outer cylinder $\left(\tau_{o}=0\right)$, i.e. equation (7) reduces to

$$
\overline{u^{\prime} w^{\prime}}-v \frac{\partial \bar{w}}{\partial r}=\frac{r_{a} \tau_{a}}{\rho r}
$$

In the DNS the no slip boundary conditions at the pipe wall are replaced by free-slip conditions, similar to the one used by [11]

$$
u(R)=0 ;\left.\frac{\partial v}{\partial r}\right|_{r=R}=\left.\frac{\partial w}{\partial r}\right|_{r=R}=0 .
$$


Fig. 11 The Reynolds shear stress $\overline{u^{\prime} w^{\prime}}$ and the total stress $\tau_{r z}=\overline{u^{\prime} w^{\prime}}-v \partial \bar{w} / \partial r$, $r_{a} / R=0.02$

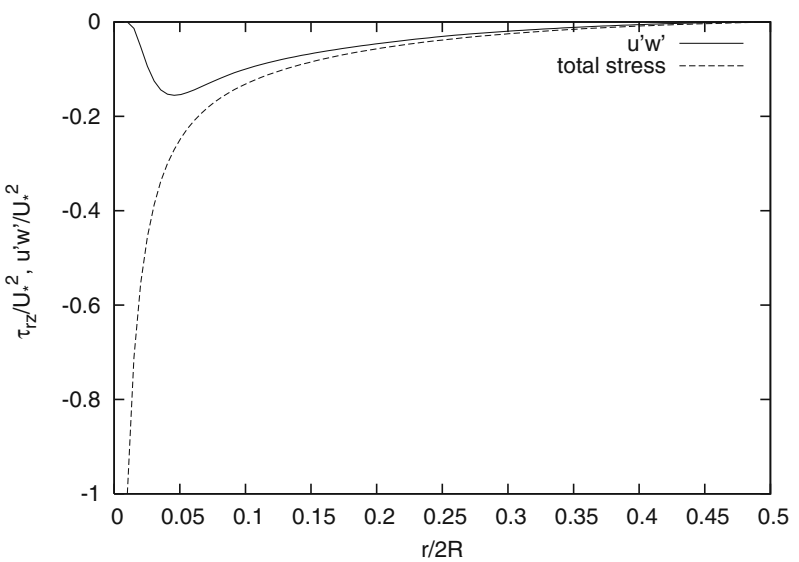

Note, that in this case we assume explicitly that the point of zero shear stress coincides with the point of maximum velocity. The flow is still driven by a constant pressure gradient $\partial \bar{p} / \partial z$. In Fig. 11 we show the Reynolds shear stress, scaled with $\sqrt{\tau_{a} / \rho}$, together with the profile given by equation (4). The value for the constant $C$ is in this case easy to calculate. At the outer boundary $r=R$ the Reynolds shear stress $\overline{u^{\prime} w^{\prime}}$ and the velocity gradient $\partial \bar{w} / \partial r$ are zero, thus the constant $C$ is equal to $\frac{1}{2 \rho} R^{2} \partial \bar{p} / \partial z$.

The mean velocity profile is shown in Fig. 12. Close to the annulus we again observe a logarithmic behavior as given by equation (9). Further away from the wall the profile given by equation (12) matches well with the DNS.

Finally, we show in Fig. 13 the rms profiles of the velocity fluctuations as a function of the non-dimensional coordinate $r^{+}=\left(r-r_{a}\right) \sqrt{\tau_{a} / \rho} / v$. The peak of the rms is considerable lower than was the case for the flow with outer pipe, see Fig. 10.

Fig. 12 The velocity profile as a function of $r^{+}=\left(r-r_{a}\right) \sqrt{\tau_{a} / \rho} / v$, scaled with with the friction velocity $\sqrt{-\tau_{a} / \rho}, r_{a} / R=0.02$

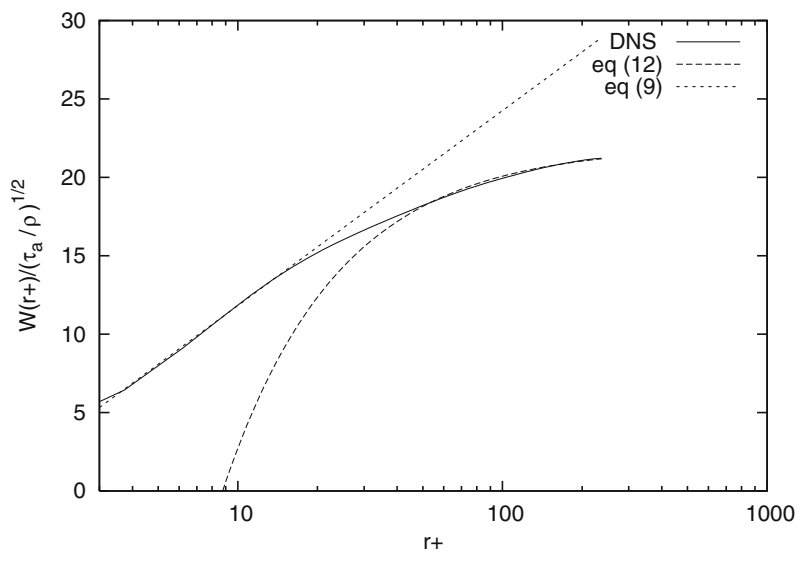


Fig. 13 The rms profile of the three velocity components as a function of $r^{+}=\left(r-r_{a}\right) \sqrt{\tau_{a} / \rho} / \nu$, scaled with the friction velocity $\sqrt{-\tau_{o} / \rho}, r_{a} / R=0.02$

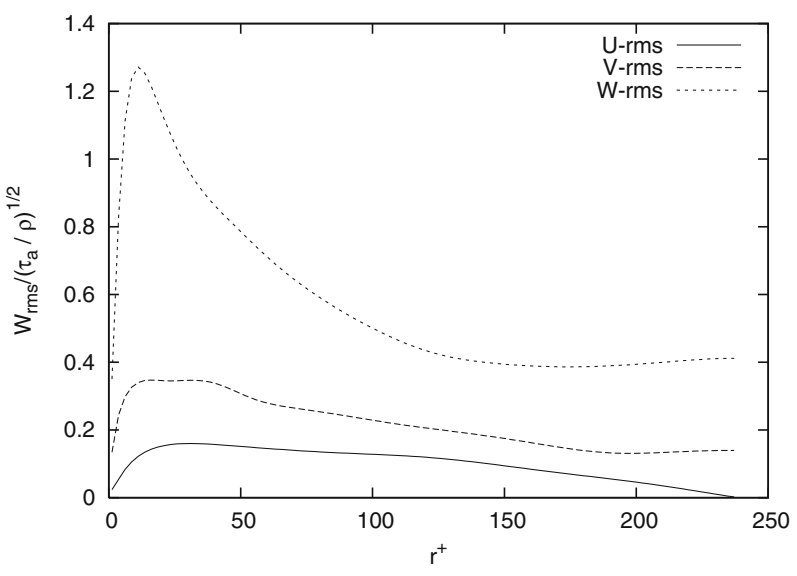

\section{Discussion and Conclusion}

In this paper we have used direct numerical simulation to calculate the flow in concentric annuli. In the literature it is argued that for this geometry the radius for which the maximum axial velocity is obtained is not the same as the position where the Reynolds shear stress is zero. If this would be the case then the widely used eddy viscosity approach would be invalid. In our direct numerical simulation we have not observed this shift between the zero shear stress point and the maximum axial velocity. This is in contrast to the DNS performed by Chung [2]. The discrepancy with the results obtained by Chung is most likely due to a poor radial resolution of this DNS. Churchill et al. use the experimental observations of Rehme [3] to support their claim. In our view the accuracy of the measured Reynolds shear stress in the work of Rehme is questionable. For the shear stress measurement he used a DISA hotwire. A slight misalignment of one degree of the crosswire could already give a faulty reading for the Reynolds shear stress. In the paper of Rehme [3] not much attention and/or documentation is given to or on the calibration of the hotwire. Furthermore, it is likely that Rehme his measurements are biased by end effects in his setup. The measurements where taken very close to the outflow of the pipe, the exact distance is not reported in the paper.

In view of our results of the DNS and the uncertainties about the experiments we feel that the argument of Churchill [7] and Rehme [3] is wrong. Unfortunately we have not yet been able to show from first principles that the points should coincide as they do in our DNS. Furthermore, we do not claim that in more general flows the point for zero shear stress should coincide with maximum velocity. An analysis for nearly parallel flows which shows this fact is given by [10].

Open Access This article is distributed under the terms of the Creative Commons Attribution Noncommercial License which permits any noncommercial use, distribution, and reproduction in any medium, provided the original author(s) and source are credited. 


\section{References}

1. Eggels, J.G.M., Unger, F., Weiss, M.H., Westerweel, J. Friedrich, R., Nieuwstadt, F.T.M.: Fully developed turbulent pipe flow: a comparison between direct numerical simulation and experiment. J. Fluid Mech. 268, 175-209 (1994)

2. Chung, S.Y., Rhee, G.h., Sung, H.J.: Direct numerical simulation of turbulent concentric annular pipe flow, Part 1: flow field. Int. J. Heat Fluid Flow 23, 426-440 (2002)

3. Rehme, K.: Turbulent flow in smooth concentric annuli with small radius ratios. J. Fluid Mech. 64, 263-287 (1974)

4. Kaneda, M., Yu, B., Ozoe, H., Churchill, S.W.: The characteristics of turbulet flow and convection in concentric circular annuli, Part I: flow. Int. J. Heat Mass Transfer 46, 5045-5057 (2003)

5. Brighton, J.A., Jones, J.B.: Fully developed turbulent flow in annuli. J. Basic Eng. 86, 835-844 (1964)

6. Quarmby, A.: An analysis of turbulent flow in concentric annuli. Appl. Sci. Res. 19, 250-273 (1968)

7. Churchill, S.W., Chan, C.: Turbulent flow in channels in terms of local turbulent shear and normal stresses. AIChE J. 41, 2513-2521 (1995)

8. Nouri, J.M., Umur, H, Whitelaw, J.H.: Flow of Newtonian and non-Newtonian fluids in concentric and eccentric annuli. J. Fluid Mech. 253, 617-641 (1993)

9. Mochizuki, S., Nieuwstadt, F.T.M.: Reynolds number-dependence of the maximum in the streamwise velocity fluctuations in wall turbulence. Exp. Fluids 21, 218-226 (1996)

10. Hinze, J.O.: Turbulent flow regions with shear stress and mean velocity gradient of opposite sign. Appl. Sci. Res. 22, 163-175 (1970)

11. Neves, J.C., Moin, P., Moser, R.D.: Effects of convex transverse curvature on wall-bounded turbulence. Part 1. The velocity and vorticity. J. Fluid Mech. 272, 349-381 (1994)

12. Pope, S.B: (2003) Turbulent Flows. Cambridge University Press 